\title{
Effect of Constitutive Material Models on Seismic Response of Two-Story Reinforced Concrete Frame
}

\author{
Md. Iftekharul Alam and Dookie Kim,*
}

(Received March 26, 2012, Accepted May 30, 2012, Published online July 1, 2012)

\begin{abstract}
This paper focuses on the finite element (FE) response sensitivity and reliability analyses considering smooth constitutive material models. A reinforced concrete frame is modeled for FE sensitivity analysis followed by direct differentiation method under both static and dynamic load cases. Later, the reliability analysis is performed to predict the seismic behavior of the frame. Displacement sensitivity discontinuities are observed along the pseudo-time axis using non-smooth concrete and reinforcing steel model under quasi-static loading. However, the smooth materials show continuity in response sensitivity at elastic to plastic transition points. The normalized sensitivity results are also used to measure the relative importance of the material parameters on the structural responses. In FE reliability analysis, the influence of smoothness behavior of reinforcing steel is carefully noticed. More efficient and reasonable reliability estimation can be achieved by using smooth material model compare with bilinear material constitutive model.
\end{abstract}

Keywords: finite element analysis, sensitivity analysis, structural reliability, constitutive models, structural response.

\section{Introduction}

The philosophy of performance-based earthquake engineering has gained recognition widely in the structural analysis and design field and has been included in many seismic design guidelines (ATC-55 2005; BSSC 2003) with noticeable advances in the field of structural reliability since last two decades. Several numerical and analytical methodologies have been developed considering the non-linear behavior, material parameters, and geometric uncertainties (Schueller et al. 2004). Der Kiureghian and Ke (1988) successfully implemented reliability methodology by using finite element (FE) method and first-order reliability method (FORM) (Ditlevsen and Madsen 1996). These methods were examined to investigate the influence of the correlation length of random property or load fields on the reliability of the subjects. The results have shown that the correlation length of property fields has influence on the displacement responses, but it may not be significant in stress limit states. The determination of design point(s) is considered as an essential step in gradient based reliability methodology. Structural response sensitivity is an important ingredient and a by-product of the design point search (Hohenbichler and Rackwitz 1986). For this purpose, the use of direct differentiation method (DDM) seems to be efficient and accurate

Department of Civil and Environmental Engineering, Kunsan National University, Kunsan 573-701, Korea. *Corresponding Author; E-mail: kim2kie@chol.com Copyright $($ The Author(s) 2012. This article is published with open access at Springerlink.com approach to perform sensitivity analysis. A method has been presented to get key sensitive attributes in the material constitutive and discrete loading parameters of force-based FE frame systems (Conte et al. 2004). The material nonlinearity was considered to get the static and dynamic responses using DDM. It was concluded that the developed procedure prone to sensitivity offers a powerful general tool for computing the responses. The analytical procedure and guidelines were developed in OpenSees (Mazzoni et al. 2005; Gu et al. 2010) framework to perform FE based reliability analysis (Haukaas and Der Kiureghian 2004). In this context, smoothed materials were modeled and the existing search algorithms were modified consequently. In case of non-linear beam column elements, response sensitivities have been evaluated with great precision using DDM (Scott et al. 2004; Scott and Filippou 2007). Similarly, the application of sensitivity and reliability analyses in the soilfoundation-structure-interaction systems has been reported in several research articles ( $\mathrm{Gu} 2008$; Gu et al. 2009a, b). Scott (2012) evaluated two existing formulations of forcebased element response sensitivity and found the consistency of first formulation, while the second one proved to be inconsistent with a high condition number.

For gradient based methods, sensitivity analysis is performed to find the design point in reliability analysis. The continuity of the obtained response is essential for most of the algorithms. The discontinuity increases computational effort and due to using improper material model the process can be interrupted. Haukaas and Der Kiureghian (2004) performed static analysis on single degree of freedom systems to explain the discontinuity effects in the displacement sensitivity results using smooth and non-smooth material 
models. The results have shown the occurrence of discontinuity at yielding and unloading points in the bi-linear material. It has been suggested that by using the smooth material models such effect can be avoided. Another study was conducted focusing on the effect of gradient discontinuities caused by non-smoothness of the material models in the reliability context (Barbato and Conte 2006). A structural system was modeled using smooth and non-smooth reinforcing steels to compare response sensitivity and reliability analysis results. The consistency of the results was fairly verified with these obtained by previous researchers. Furthermore, study on the dynamic analysis concluded that the discontinuities can be effectively eliminated by providing smoothing effect in reinforcing steel and refining the time discretization of the equations of motion.

This paper presents the modeling and analysis of a reinforced concrete (RC) structure using different concrete and steel constitutive material models in the context of sensitivity based reliability analysis. DDM based response sensitivity analysis has been conducted for smooth and nonsmooth material constitutive laws. Quasi-static cyclic analysis results show that discontinuities arise in the response sensitivities while using non-smooth material models. Moreover, this difficulty can be eliminated by using smooth constitutive material models. Later, the smoothness effect of reinforcing steel on failure probabilities of frame structure has been observed using structural reliability analysis.

\section{Material Models}

Different concrete and steel material constitutive models are used to describe the structural behavior considering smoothness effect of the materials. The Kent-Scott-Park (Scott et al. 1982) concrete model is adopted as zero tension and convenient for application in engineering for its simple form. While Smoothed Popovics-Saenz (Balan et al. 1997, 2001; Zona et al. 2005) concrete also modeled with zero tension stiffening having smoothed skeleton curve. In FE structural modeling, fiber concrete sections using confined and unconfined concrete layer are considered. Figures 1 and 2 show the typical comparison between two concrete models. In case of steel material, 1D $J_{2}$ plasticity model (Conte et al. 2003) (also known as bi-linear inelastic model) and smooth inelastic Menegotto-Pinto (M-P) model (Menegotto and Pinto 1973) are used in material modeling of the structure as shown in Fig. 3. Bi-linear model with pure kinematic hardening, $H_{k i n}$ (zero isotropic hardening, $H_{\text {iso }}$ ) is considered. As a non-smooth plasticity model, $J_{2}$ plasticity model with Von Mises yield surface is well-known for metallic materials. This model has shown discontinuities in the responses when the material enters the plastic regime (Conte 2001). The version of M-P model extended by Filippou et al. (1983) has been considered to account for the isotropic strain hardening. Figure 3 shows that yield stress, $f_{y}$ and initial elastic tangent (modulus of elasticity), $E$ for both of the steel models are same. Post yield tangent

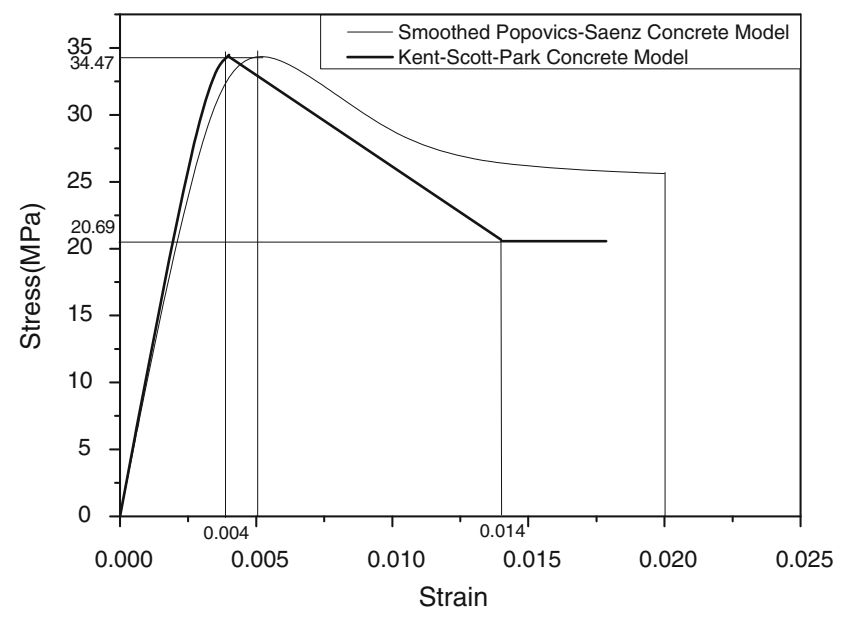

Fig. 1 Comparison between two confined concrete models.

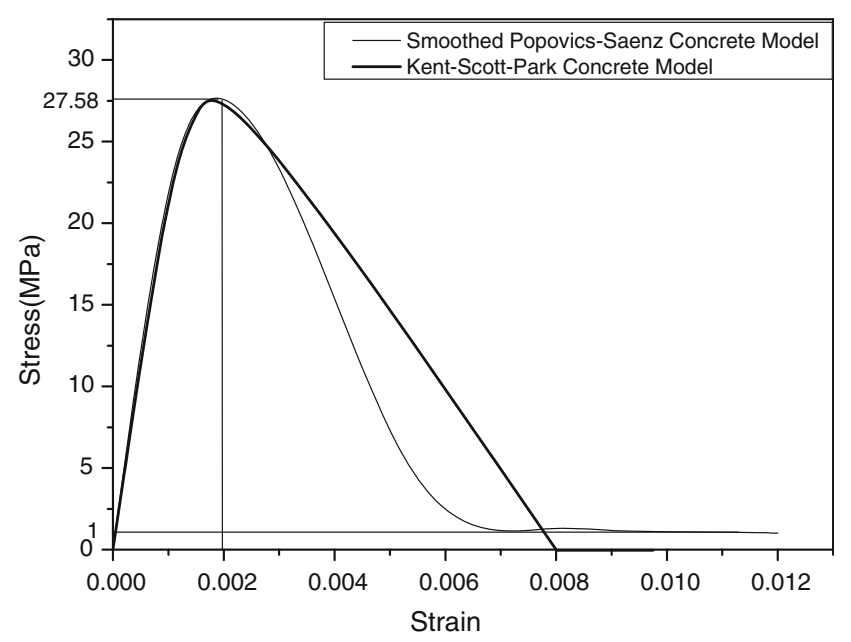

Fig. 2 Comparison between two unconfined concrete models.

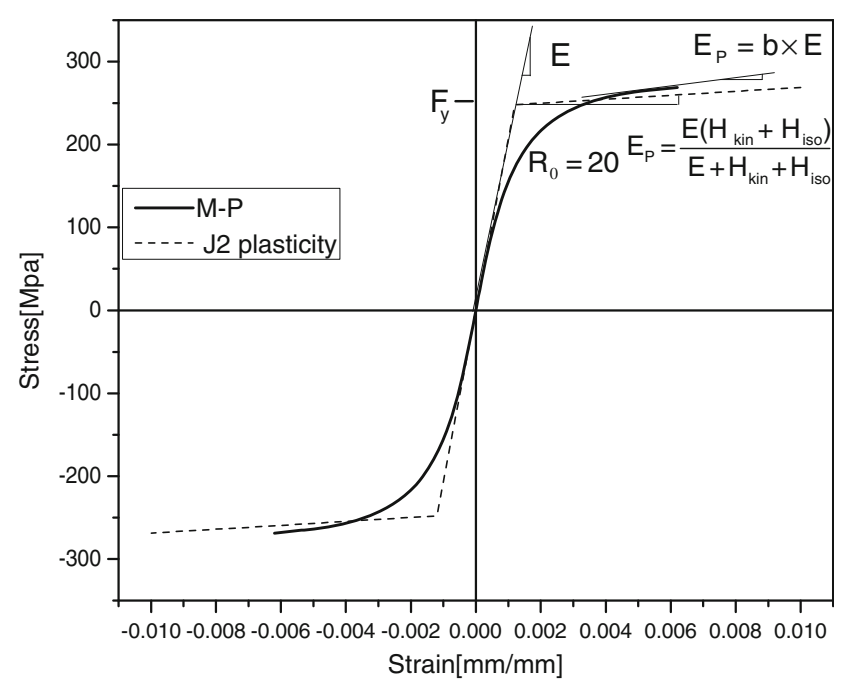

Fig. 3 Typical stress-strain curves of different constitutive steel models.

modulus for $J_{2}$ plasticity model and M-P model can be expressed by the Eqs. (1) and (2) as follows:

$$
E_{P}=\frac{E\left(H_{\text {kin }}+H_{\text {iso }}\right)}{E+H_{\text {kin }}+H_{\text {iso }}}
$$




$$
E_{P}=b \times E
$$

where $b$ is known as strain-hardening ratio and $\mathrm{R}_{0}$ controls the transition from elastic to plastic regions (Fig. 3). The M-P 1D plasticity model has shown good agreement with the experimental results, particularly for cyclic tests on reinforcing steel bars. It is observed that the constitutive law of $\mathrm{M}-\mathrm{P}$ model is smooth and continuously differentiable with respect to constitutive material parameters, thus response sensitivities continuous everywhere. Local buckling effect of steel bars in RC members can be taken into account by further modifications in M-P model (Monti and Nuti 1992).

\section{DDM Based Sensitivity Analysis}

FE response sensitivity analysis is an important analytical tool in structural optimization, FE model updating, reliability analysis, and structural identification (Ditlevsen and Madsen 1996; Kleiber et al. 1997). The response sensitivity is a measure of the change in the response quantity due to a unit change in a system parameter. Cross-sectional geometry, materials properties, applied loads or nodal co-ordinates are considered as the key parameters. Generally, the structural response can be characterized in terms of deformations, forces, or integrated quantities, i.e., dissipated energy and accumulated damage (Haukaas and Der Kiureghian 2004). Such response can be computed by structural analysis tools such as OpenSees. The DDM method is well known for computing response quantities with high level of accuracy (Kleiber et al. 1997; Arora and Haug 1979; Zhang and Der Kiureghian 1993). In nonlinear DDM based analysis structural response is calculated in each time step after achieving the convergence of the response computation. Differentiation of the algorithm is required to obtain the response using specific sensitivity parameter.

The equations of the response sensitivities were derived by Zhang and Der Kiureghian (1993). After spatial discretization using the FE method, the equation of motion of the considered system can be expressed as (Gu et al. 2009b):

$$
\boldsymbol{M}(\theta) \ddot{\boldsymbol{u}}(t, \theta)+\boldsymbol{C}(\theta) \dot{\boldsymbol{u}}(t, \theta)+\boldsymbol{R}[\boldsymbol{u}(t, \theta), \theta]=\boldsymbol{F}(t, \theta)
$$

where $t$ is the time, $\theta$ is the scalar sensitivity parameter as a material constitutive parameter, $\boldsymbol{u}$ is the vector of nodal displacements, $\boldsymbol{M}$ is the mass matrix, $\boldsymbol{C}$ is the damping matrix, $\boldsymbol{R}$ is the history dependent internal (inelastic) resisting force vector, and $\boldsymbol{F}$ is the applied dynamic load vector.

Newmark- $\beta$ method is used for the numerical integration of Eq. (3). The dynamic residual $\Psi\left(\boldsymbol{u}_{n+1}\right)$ can be written at discrete time $t=t_{n+1}=(n+1) \Delta t$ (where $\Delta t$ is the constant integration time step) as follows:

$$
\begin{aligned}
& \Psi\left(\boldsymbol{u}_{n+1}\right) \\
& \quad=\tilde{\boldsymbol{F}}_{n+1}-\left[\frac{1}{\beta(\Delta t)^{2}} \boldsymbol{M} \boldsymbol{u}_{n+1}+\frac{\alpha}{\beta(\Delta t)} \boldsymbol{C} \boldsymbol{u}_{n+1}+\boldsymbol{R}\left(\boldsymbol{u}_{n+1}\right)\right] \\
& \quad=0
\end{aligned}
$$

$\tilde{\boldsymbol{F}}_{n+1}$ can be expressed as below:

$$
\begin{aligned}
\tilde{\boldsymbol{F}}_{n+1}= & \boldsymbol{F}_{n+1} \\
& +\boldsymbol{M}\left[\frac{1}{\beta(\Delta t)^{2}} \boldsymbol{u}_{n}+\frac{1}{\beta(\Delta t)} \dot{\boldsymbol{u}}_{n}-\left(1-\frac{1}{2 \beta}\right) \ddot{\boldsymbol{u}}_{n}\right] \\
& +\boldsymbol{C}\left[\frac{\alpha}{\beta(\Delta t)} \boldsymbol{u}_{n}-\left(1-\frac{\alpha}{\beta}\right) \dot{\boldsymbol{u}}_{n}-(\Delta t)\left(1-\frac{\alpha}{2 \beta}\right) \ddot{\boldsymbol{u}}_{n}\right]
\end{aligned}
$$

Differentiating Eq. (4) with respect to $\theta$,

$$
\begin{gathered}
{\left[\frac{1}{\beta(\Delta t)^{2}} \boldsymbol{M}+\frac{\alpha}{\beta(\Delta t)} \boldsymbol{C}+\left(\boldsymbol{K}_{T}^{s t a t}\right)_{n+1}\right] \frac{\partial \boldsymbol{u}_{n+1}}{\partial \theta}} \\
=-\left(\frac{1}{\beta(\Delta t)^{2}} \frac{\partial \boldsymbol{M}}{\partial \theta}+\frac{\alpha}{\beta(\Delta t)} \frac{\partial \boldsymbol{C}}{\partial \theta}\right)_{\boldsymbol{u}_{n+1}} \\
\quad-\left.\frac{\partial \boldsymbol{R}\left(\boldsymbol{u}_{n+1}(\theta), \theta\right)}{\partial \theta}\right|_{\boldsymbol{u}_{n+1}}+\frac{\partial \tilde{\boldsymbol{F}}_{n+1}}{\partial \theta}
\end{gathered}
$$

here

$$
\begin{aligned}
& \frac{\partial \tilde{\boldsymbol{F}}_{n+1}}{\partial \theta} \\
& =\frac{\partial \boldsymbol{F}_{n+1}}{\partial \theta}+\frac{\partial \boldsymbol{M}}{\partial \theta}\left(\frac{1}{\beta(\Delta t)^{2}} \boldsymbol{u}_{n}+\frac{1}{\beta(\Delta t)} \dot{\boldsymbol{u}}_{n}-\left(1-\frac{1}{2 \beta}\right) \ddot{\boldsymbol{u}}_{n}\right) \\
& \quad+\boldsymbol{M}\left[\frac{1}{\beta(\Delta t)^{2}} \frac{\partial \boldsymbol{u}_{n}}{\partial \theta}+\frac{1}{\beta(\Delta t)} \frac{\partial \dot{\boldsymbol{u}}_{n}}{\partial \theta}-\left(1-\frac{1}{2 \beta}\right) \frac{\partial \ddot{\boldsymbol{u}}_{n}}{\partial \theta}\right] \\
& \quad+\frac{\partial \boldsymbol{C}}{\partial \theta}\left[\frac{\alpha}{\beta(\Delta t)} \boldsymbol{u}_{n}-\left(1-\frac{\alpha}{\beta}\right) \dot{\boldsymbol{u}}_{n}-(\Delta t)\left(1-\frac{\alpha}{2 \beta}\right) \ddot{\boldsymbol{u}}_{n}\right] \\
& \quad+\boldsymbol{C}\left[\frac{\alpha}{\beta(\Delta t)} \frac{\partial \boldsymbol{u}_{n}}{\partial \theta}-\left(1-\frac{\alpha}{\beta}\right) \frac{\partial \dot{\boldsymbol{u}}_{n}}{\partial \theta}-(\Delta t)\left(1-\frac{\alpha}{2 \beta}\right) \frac{\partial \ddot{\boldsymbol{u}}_{n}}{\partial \theta}\right]
\end{aligned}
$$

In Eq. (5) the partial derivative of the internal resisting force vector $\boldsymbol{R}\left(\boldsymbol{u}_{n+1}\right)$ with respect to sensitivity parameter $\theta$ can be presented by the term $\left[\left.\partial \boldsymbol{R}\left(\boldsymbol{u}_{n+1}(\theta), \theta / \partial \theta\right]\right|_{\boldsymbol{u}_{n+1}}\right.$ where the displacement vector $\boldsymbol{u}_{n+1}$ remains fixed.

In Eq. (5) $\boldsymbol{K}_{T}^{d y n}=1 / \beta(\Delta t)^{2} \boldsymbol{M}+\alpha / \beta(\Delta t) \boldsymbol{C}+\left(\boldsymbol{K}_{T}^{\text {stat }}\right)_{n+1}$ represents the tangent dynamic stiffness matrix, where, $\left(\boldsymbol{K}_{T}^{\text {stat }}\right)_{n+1}^{i}$ is the consistent tangent stiffness matrix defined by $\left(\boldsymbol{K}_{T}^{\text {stat }}\right)_{n+1}=\partial \boldsymbol{R}_{n+1} / \partial \boldsymbol{u}_{n+1}$. The word 'consistent' emphasizes that the tangent operator is obtained through consistent linearization of the constitutive law integration scheme, which guarantees the quadratic rate of asymptotic convergence of the iterative solution strategies based on Newton's method (Simo and Taylor 1985).

\section{FE Structural Model}

The present study consists of modeling and analysis of a RC frame (Fig. 4) subjected to earthquake loading using OpenSees. The structure is modeled using the displacement-based, Euler-Bernoulli frame element with the distributed plasticity (Gu et al. 2010). In each element five integration points are provided and section stress results are computed by discretizing the frame sections into layer. Two different constitutive 


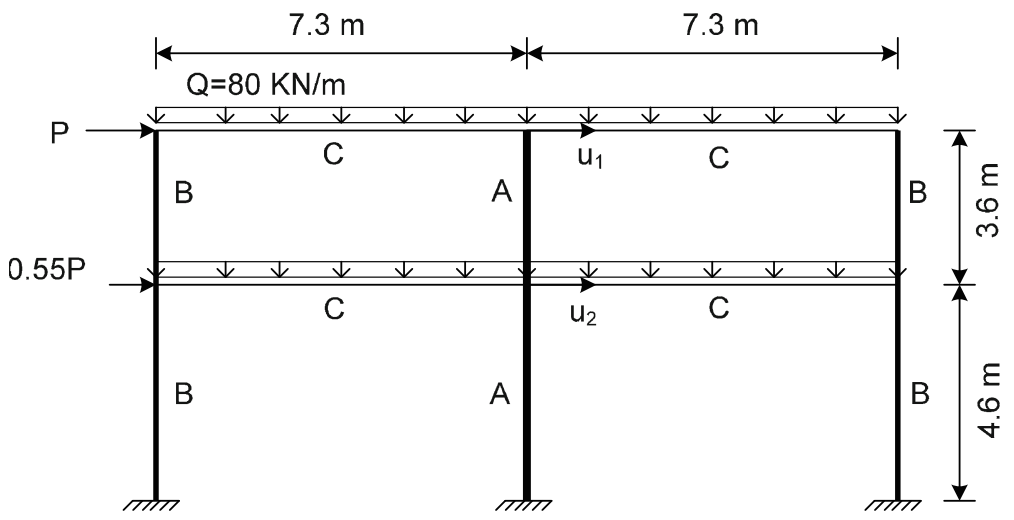

Fiber-discretized cross-sections (unit: $\mathrm{cm}$ )

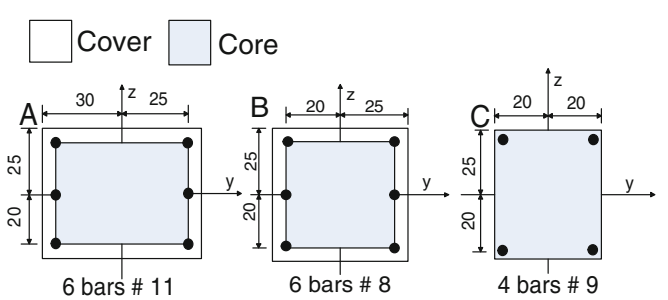

$A=$ Interior column section

$\mathrm{B}=$ Exterior column section

C $=$ Girder section

Fig. 4 Geometry, loading due to gravity and cross-sectional properties of frame structure.

concrete models are used to evaluate the response sensitivity of the material with the smoothing effect. The parameters used are: $f_{c}=$ concrete compressive strength; $f_{u}=$ concrete crushing strength; $\varepsilon_{0}=$ concrete strain at maximum strength; $\varepsilon_{u}=$ concrete strain at crushing strength; $E_{c}=$ initial tangent stiffness; and $\eta=$ smoothing parameter. In the column section, different material parameters are used for the unconfined and confined concrete parts. In case of steel material, 1D $J_{2}$ plasticity and M-P models are considered. The steel parameters used as: $E=$ Young's modulus; $f_{y}=$ yield strength; $H_{\text {kin }}=$ kinematic hardening modulus; $H_{\text {iso }}=$ isotropic hardening modulus; $b=$ strain-hardening ratio, $R_{0}, c R_{1}$, $c R_{2}=$ parameters which control the elastic to plastic transition; $a_{1}, a_{2}=$ isotropic hardening parameters. The input parametric values being used are listed in the Table 1 and 2 . The dead and live loads are applied in the frame using lumped mass phenomenon at the nodal points.

The cyclic loading and dynamic base excitation are applied on the 2D frame to get response sensitivities. The lateral forces are applied at the corresponding nodes of each floor level as shown in Fig. 4. The total horizontal base acceleration used in the dynamic analysis (Fig. 5) is scaled by 10 with peak ground acceleration $14.89 \mathrm{~m} / \mathrm{s}^{2}$ (Zhang et al. 2008). Newmark- $\beta$ method is used for the integration operations with parameters $\beta=0.2756$ and $\gamma=0.55$ with a constant time interval $\Delta t=0.01[\mathrm{~s}](\mathrm{Gu}$ et al. 2009a). Response sensitivities are calculated for confined and unconfined concrete and reinforcing steel parameters by DDM as well as forward finite difference (FFD) method.
Table 2 Parametric values of different steel constitutive models.

\begin{tabular}{c|c|c|c}
\hline \multicolumn{2}{c|}{ M-P model } & \multicolumn{2}{c}{$J_{2}$ plasticity model } \\
\hline \hline$E(\mathrm{kPa})$ & $2.1 \times 10^{8}$ & $E(\mathrm{kPa})$ & $2.1 \times 10^{8}$ \\
\hline$f_{y}(\mathrm{kPa})$ & $2.48 \times 10^{5}$ & $f_{y}(\mathrm{kPa})$ & $2.48 \times 10^{5}$ \\
\hline$b$ & 0.02 & $H_{\text {kin }}(\mathrm{kPa})$ & $1.6129 \times 10^{6}$ \\
\hline$R_{0}$ & 20 & $H_{\text {iso }}$ & 0.0 \\
\hline$c R_{1}$ & 0.925 & & \\
\hline$c R_{2}$ & 0.15 & & \\
\hline$a_{1}$ & 18.5 & & \\
\hline$a_{2}$ & 0.15 & & \\
\hline
\end{tabular}

FE reliability method is used to estimate probabilities of failure to achieve predefined performance. The performance functions are defined in terms of lateral displacement response of frame structure. For a simplest case of one performance function, the reliability problem can be defined as (Haukaas and Der Kiureghian 2004)

$$
p_{f}=\int_{g(x) \leq 0} f(x) d x
$$

where $p_{f}$ is the failure probability, $x$ is the FE model parameters vector, $g(x)$ is the performance function and $f(x)$ is the joint probability density function of $x$. An approximate solution of the above integral equation can be

Table 1 Parametric values of different concrete constitutive models.

\begin{tabular}{c|c|c|c|c|c}
\hline \multicolumn{2}{c|}{ Smoothed Popovics-Saenz concrete model } & \multicolumn{3}{c}{ Kent-Scott-Park concrete model } \\
\hline \hline Parameter & Core concrete & Cover concrete & Parameter & Core concrete & Cover concrete \\
\hline$f_{c}(\mathrm{kPa})$ & 34473.8 & 27579.04 & $f_{c}(\mathrm{kPa})$ & 34485.6 & 27588.5 \\
\hline$f_{u}(\mathrm{kPa})$ & 25723.0 & 1000.0 & $f_{u}(\mathrm{kPa})$ & 20691.4 & 0.0 \\
\hline$\varepsilon_{0}$ & 0.005 & 0.002 & $\varepsilon_{0}$ & 0.004 & 0.002 \\
\hline$\varepsilon_{u}$ & 0.02 & 0.012 & $\varepsilon_{u}$ & 0.014 & 0.008 \\
\hline$E_{c}(\mathrm{kPa})$ & $2.7851 \times 10^{7}$ & $2.4910 \times 10^{7}$ & & & \\
\hline$\eta$ & 0.2 & 0.2 & & & \\
\hline
\end{tabular}




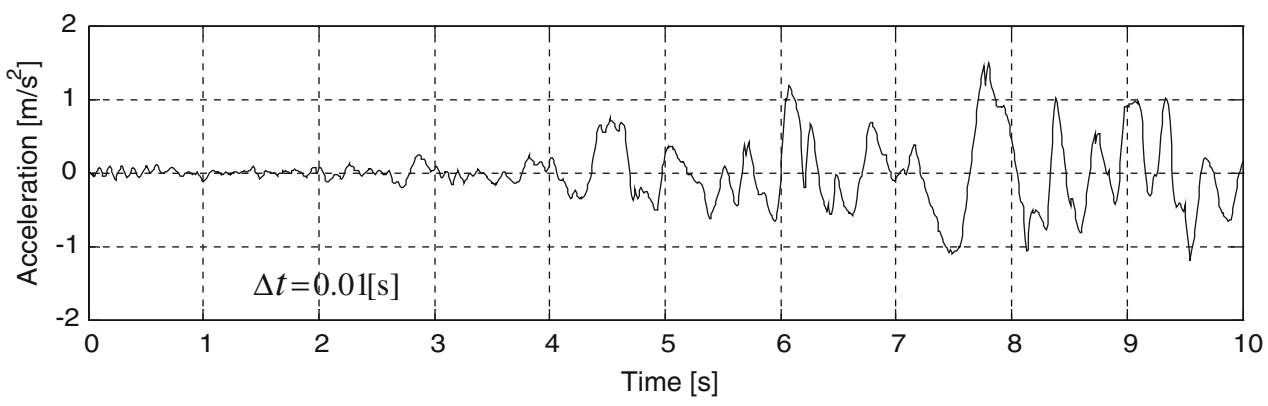

Fig. 5 Acceleration time history at the base of the RC frame.

done by various available methods (e.g., FORM, sampling analysis). Advanced reliability method (FORM), and important sampling method are used to obtain failure probability and Hasofer-Lind (Hasofer and Lind 1974) reliability index $\beta$ of frame structure. After the completion of the sensitivity analysis a static pushover study is carried out to evaluate the structural reliability performance. Applied lateral pushover load is same as the quasi-static cyclic loading. Smoothed Popovics-Saenz model has been chosen for further numerical study due to better performance in sensitivity analysis. All concrete and steel material parameters are characterized as random variables (RVs). All material RVs with lognormal distributions and probabilistic properties are listed in Table 3. Table 4 indicates the correlation coefficient between the RVs for each pair of members. The values of all RVs have been considered from another study (Gu 2008).

Two different limit state functions (LSF) are considered:

$$
\begin{aligned}
& g_{1}=\Delta_{\lim }-u_{1} \\
& g_{2}=u_{\lim }-\left(u_{1}-u_{2}\right)
\end{aligned}
$$

The first LSF $\left(g_{1}\right)$ defines the failure event as the exceedance of the lateral roof displacement from 1 to $2 \%$ of the building height, while the second LSF $\left(g_{2}\right)$ defines a similar threshold for inter-story drift (Haukaas and Der Kiureghian 2004). Here, $\Delta_{\lim }=1-2 \%$ of the building height, defines the first critical collapse displacement; $u_{\text {lim }}=1-2 \%$ of the interstory height is second critical collapse displacement; $u_{1}=$ roof displacement; and $\left(u_{1}-u_{2}\right)=$ inter-story drift. The freeware reliability tools called Risk tools (Rt) software is used to compute the structural reliability directly by using LSF equations (Mahsuli and Haukaas 2010). The OpenSees FE model is imported to Rt software, then all the RVs,
Table 4 Correlation between the RVs.

\begin{tabular}{c|c|c}
\hline $\mathrm{RV}$ & $\mathrm{RV}$ & Correlation \\
\hline \hline$f_{c(\text { core })}$ & $f_{c(\text { cover })}$ & 0.8 \\
\hline$\varepsilon_{0(\text { core })}$ & $\varepsilon_{0(\text { cover })}$ & 0.8 \\
\hline$\varepsilon_{u(\text { core })}$ & $\varepsilon_{\text {u(cover })}$ & 0.8 \\
\hline$f_{c(\text { core })}$ & $f_{u(\text { core })}$ & 0.8 \\
\hline$\varepsilon_{0(\text { core })}$ & $\varepsilon_{u(\text { core })}$ & 0.8 \\
\hline$\varepsilon_{0(\text { cover })}$ & $\varepsilon_{\mathrm{u}(\text { cover })}$ & 0.8 \\
\hline$\varepsilon_{0(\text { core })}$ & $\varepsilon_{\mathrm{u}(\text { cover })}$ & 0.64 \\
\hline$f_{c(\text { core })}$ & $\varepsilon_{0(\text { cover })}$ & 0.64 \\
\hline$f_{u(\text { core })}$ & $f_{u(\text { cover })}$ & 0.64 \\
\hline
\end{tabular}

their mean, standard deviation and correlation factors are inputted. Then, based on the failure functions (LSF), corresponding failure probability and reliability index are computed within Rt software framework.

\section{Results and Discussion}

From the quasi-static cyclic analysis, the results are shown in Figs. 6 and 7. It is observed that, Popovics-Saenz concrete model with smoothing parameter $(\eta)$ shows smooth continuous loading-unloading displacement response sensitivity curve under quasi-static cyclic loading (Fig. 6). On the other hand, the discontinuities in the response sensitivities are clearly visible from Popovics-Saenz concrete model without smoothing parameter and Kent-Scott-Park concrete model. The value of eta is used as 0.2 and obtained from the

Table 3 Probabilistic properties of RC frame structure materials.

\begin{tabular}{c|c|c|c|c|c|c|c}
\hline & \multicolumn{4}{|c|}{ Concrete } & & \multicolumn{2}{c}{ Steel } \\
\cline { 2 - 7 } Material & Mean & $\begin{array}{c}\text { Coefficient of } \\
\text { variation }\end{array}$ & Mean & $\begin{array}{c}\text { Coefficient of } \\
\text { variation }\end{array}$ & & $\begin{array}{c}\text { Mean } \\
\text { Coefficient of } \\
\text { variation }\end{array}$ \\
\hline \hline$f_{c}(\mathrm{kPa})$ & 34473.8 & 0.2 & 27579.04 & 0.2 & $E(\mathrm{kPa})$ & $2.1 \times 108$ & 0.033 \\
\hline$f_{u}(\mathrm{kPa})$ & 25723.0 & 0.2 & 1000.0 & - & $f_{y}(\mathrm{kPa})$ & $2.48 \times 105$ & 0.106 \\
\hline$\varepsilon_{0}$ & 0.005 & 0.2 & 0.002 & 0.2 & $b$ & 0.02 & 0.2 \\
\hline$\varepsilon_{u}$ & 0.02 & 0.2 & 0.012 & 0.2 & & & \\
\hline
\end{tabular}



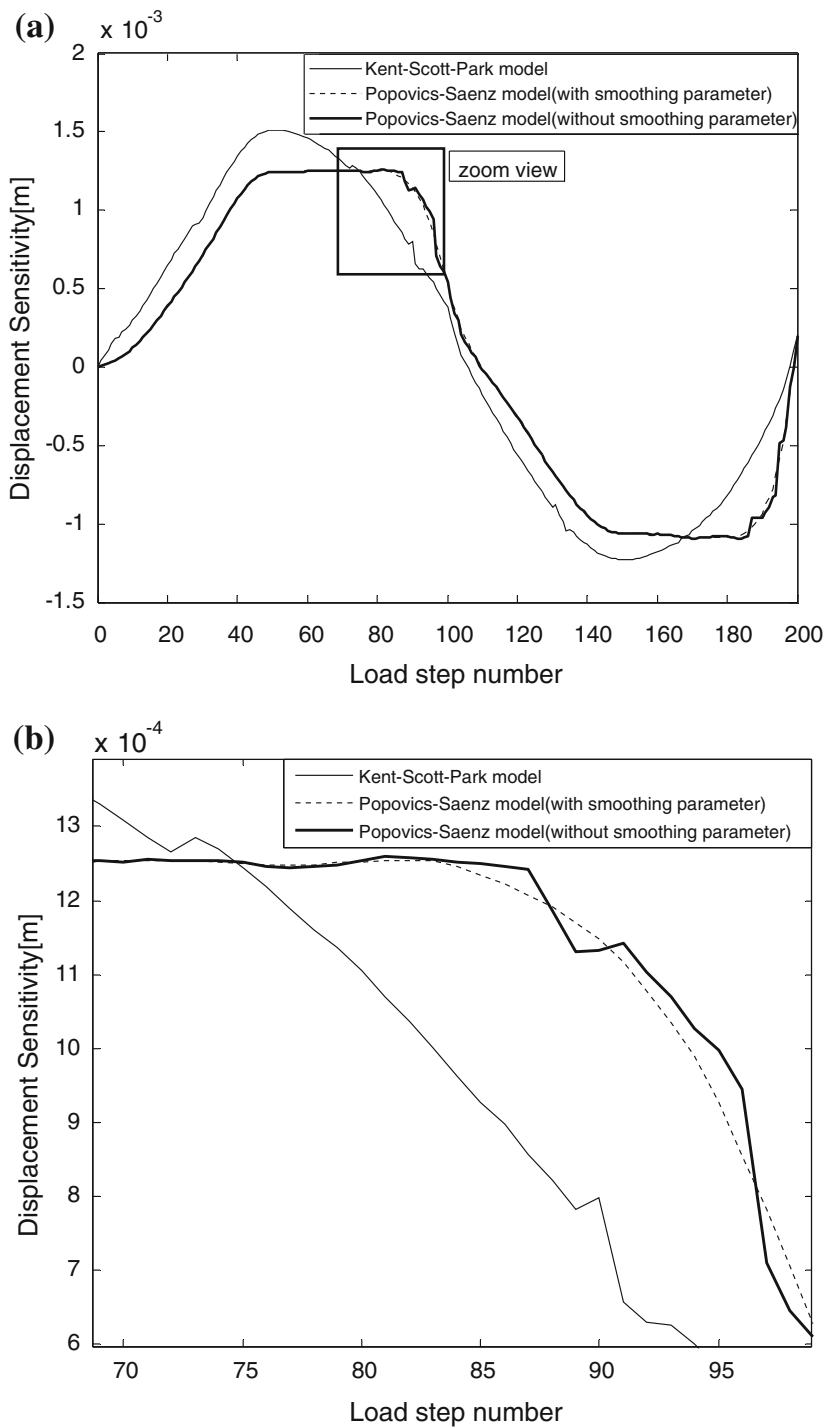

Fig. 6 a Displacement sensitivity with respect to core concrete compressive stress, $f_{c}$ under static loading. b Zoom view.

previous study (Gu et al. 2010). Thus the smoothing parameter of Popovics-Saenz concrete model has a considerable contribution to achieve continuous response sensitivity in concrete constitutive model. For bi-linear steel $\left(J_{2}\right.$ plasticity) model response sensitivities are discontinuous at the elastic-to-plastic transition points whereas, they are continuous throughout the curve for smooth steel model (Fig. 7). Previous findings by some researchers also indicate the same phenomenon in the similar manner (Haukaas and Der Kiureghian 2004; Barbato and Conte 2006). Thus, the continuity of the response computation can be achieved by using smooth material models which is essential for the convergence in the search of design point in reliability analysis.

For the validation case, smoothed Popovics-Saenz concrete and $\mathrm{M}-\mathrm{P}$ steel are used in 2D frame structure and earthquake motion is applied. Here the comparison between DDM and FFD method outcomes are shown for two material parameters. In first case, sensitivity of the lateral roof displacement due to the yield strength of reinforcing steel $f_{y}$ is presented in Fig. 8 .
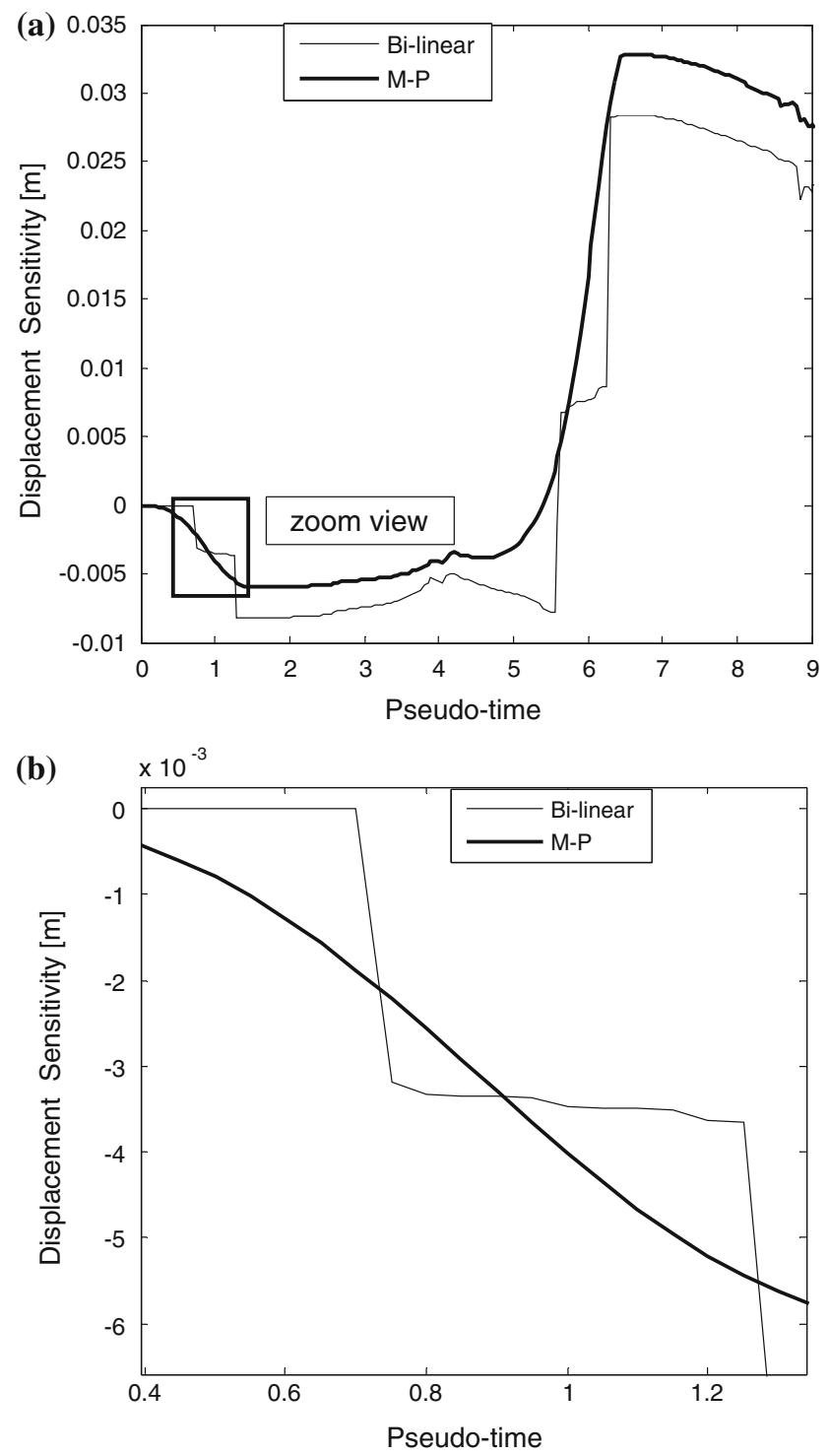

Fig. 7 a Displacement sensitivity with respect to steel Young's modulus, $E$ under static loading. b Zoom view.

Three perturbation levels are considered, namely FFD 00.01 , FFD 0.001, FFD 0.0001. Compressive strength of confined concrete, $f_{c}$ is considered as the second parameter to validate the results (Fig. 9) and three levels of perturbation are considered as before. In both cases, FFD results converge well to the DDM results and the FFD results for perturbation level 0.0001 are close to the DDM results.

Figure 10 shows the displacement time history of the models used in this study. For the Kent-Scott-Park concrete model, maximum roof displacement to core concrete compressive strength $f_{c}$ is more than that of smoothed Popovics-Saenz model. In both cases $\mathrm{M}-\mathrm{P}$ reinforcing steel model has been used. It is inferred that under dynamic motions displacement responses of both concrete models are smooth along the time axis due to the inertia effects. Both of the models exhibit large plastic deformations under dynamic loading.

The structure is then modeled with $\mathrm{M}-\mathrm{P}$ and $J_{2}$ plasticity reinforcing steel models. Figure 11 demonstrates the computed time histories of the inter-story drift for two different steel models. Results corresponding to M-P model are 

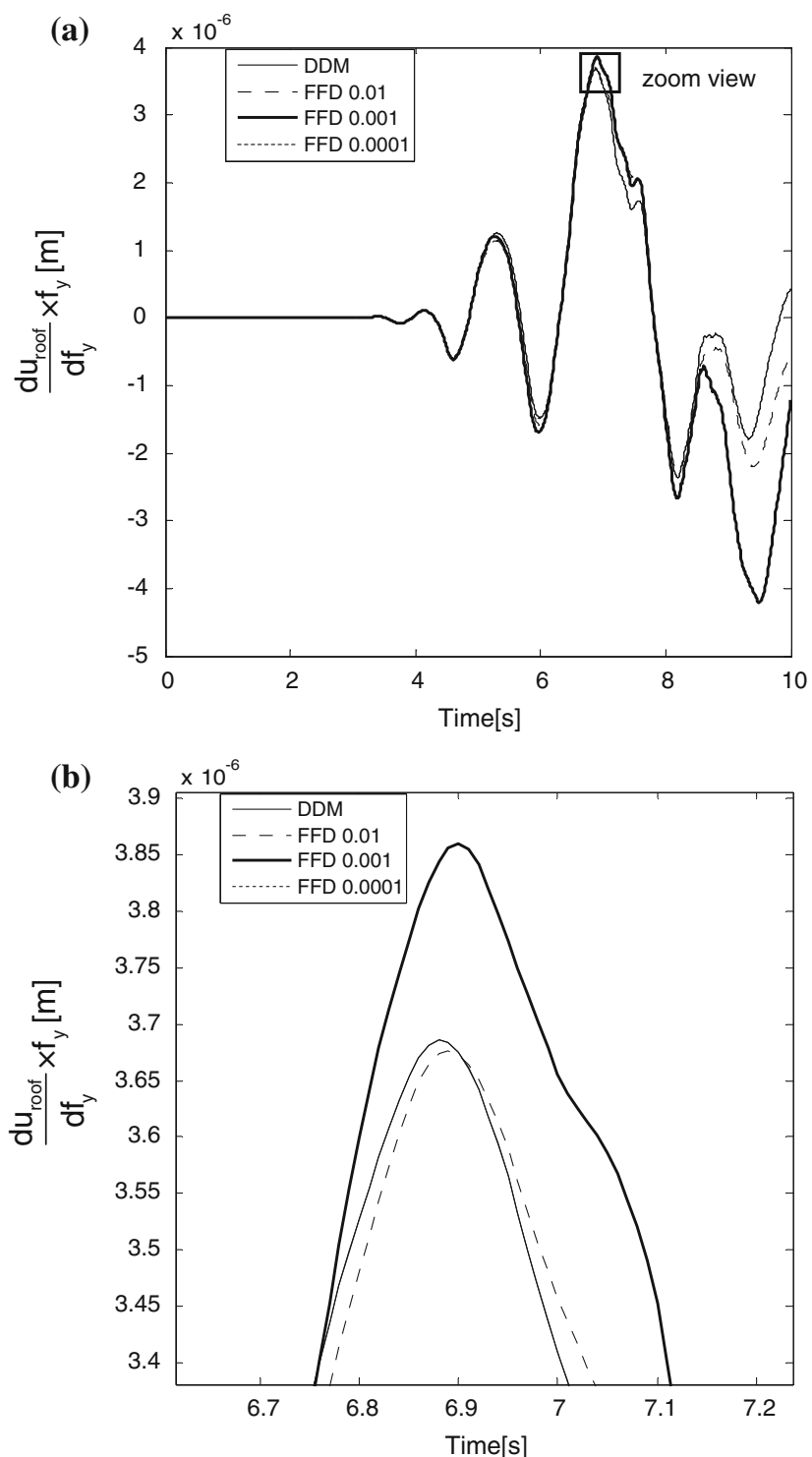

Fig. 8 a Validation of DDM results by FFD analysis: sensitivity of roof horizontal displacement response to the yield strength, $f_{y}$ of steel. b Zoom view.

not found very close to the ones obtained from the $J_{2}$ plasticity model. It is also noticed that the maximum displacement occurs while using $J_{2}$ plasticity model. Large plastic deformations are also observed at several points of constitutive steel models.

Figure 12 displays the sensitivity response of the lateral roof displacement with respect to the yield strength, $f_{y}$ and steel Young's modulus, $E$ under dynamic action. No discontinuities are found in the response sensitivities along the time axis for both steel models. Thus, the effect of inertia in the sensitivity equation is responsible to eliminate the discontinuities (Haukaas and Der Kiureghian 2004). Moreover, from the sensitivity results along the parameter axis, a different behavior is found in terms of discontinuity of the curves. Figure 12a, b shows small magnitude of discontinuities for response sensitivities obtained from the nonsmooth $J_{2}$ plasticity model, on the other hand M-P models are quite smoothen along the parameter axis. Table 5 shows the importance ranking of the material parameters. The
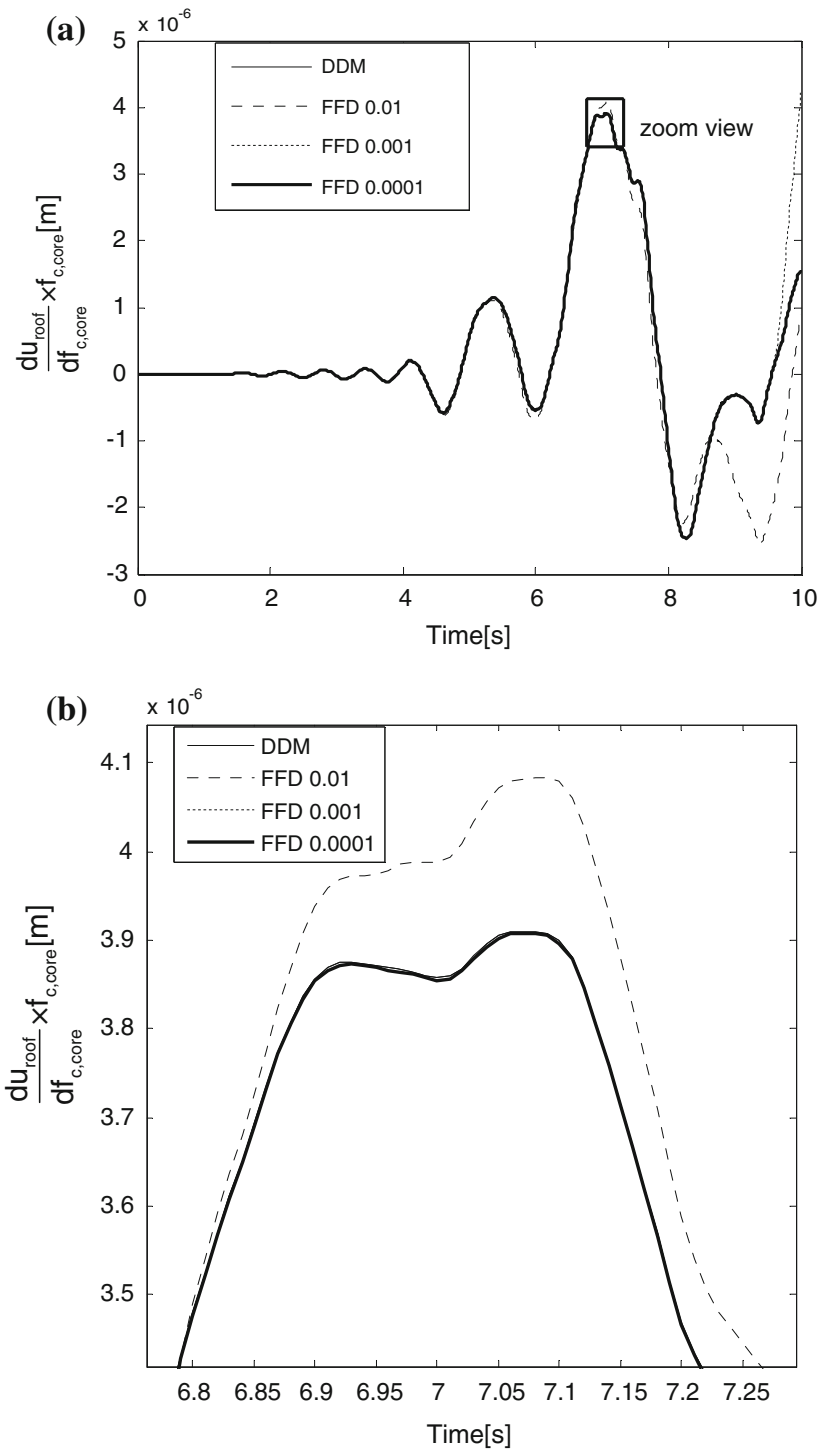

Fig. 9 a Validation of DDM results by FFD analysis: sensitivity of roof horizontal displacement response to the core concrete strength parameter, $f_{c}$. b Zoom view.

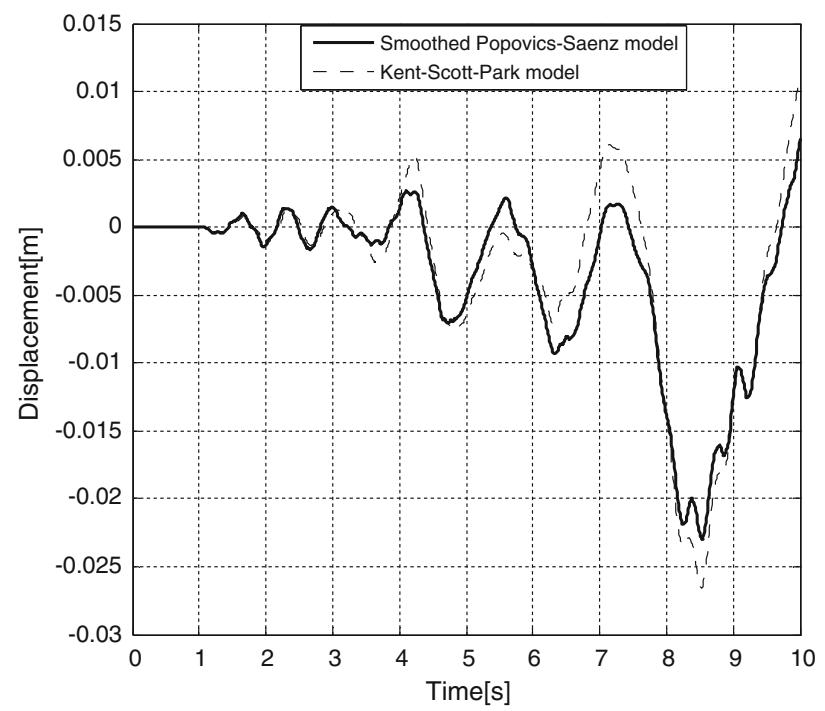

Fig. 10 Roof displacement responses due to core concrete compressive strength $f_{c}$ of two different concrete models. 


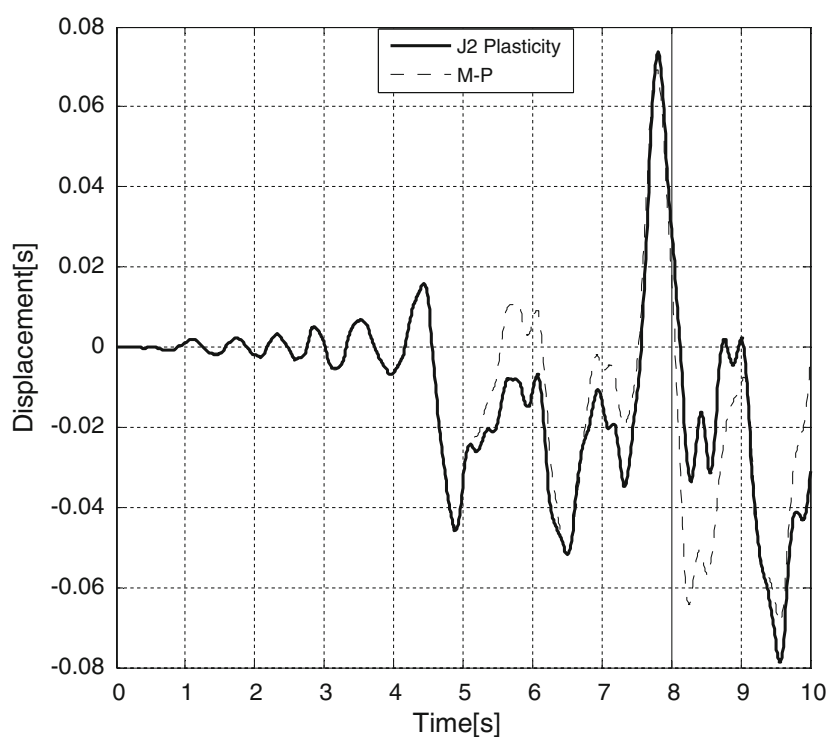

Fig. 11 Response histories of inter-story drift for different constitutive steel models.

normalized response sensitivity results for the inter-story drift $u_{\text {drift }}=u_{1}-u_{2}$ are used to determine the significance of material parameters. Smoothed Popovics-Saenz concrete model and M-P steel model are used to select the effectiveness of material parameters. It is observed that parameter $E, f_{y}, f_{c}$ are considered to be most important in earthquake excitations.

Reliability analysis results for the frame are also evaluated considering material parameters as RVs. Important sampling and FORM analyses are conducted for two LSF. Results in Table 6 show good agreement between two methods which indicate the accuracy of FORM result in OpenSees framework. The outcomes also reflected that Reliability index $\beta$ for LSF $g_{1}$ is more than LSF $g_{2}$. Thus, the structure is more reliable with respect to roof displacement rather than interstory drift limit. Figures 13 and 14 present the CDF for the roof displacement and the inter-story drift of the frame using FORM analysis. At each point of the CDF curve one analysis run is required. The values of failure probabilities are obtained by changing the first and second critical collapse displacement values within a range of 1-2\% building height and inter-story height respectively, in Eqs. (7) and (8). The CDF curves of smooth (M-P) steel material are slightly different from non-smooth ( $J_{2}$ plasticity) material in both cases. For $1.1 \%$ of the roof displacement threshold, corresponding probabilities of failure for smoothed and nonsmoothed material are 0.99976 and 0.986574 respectively. From CDF of the inter-story drift, very similar failure probabilities with that of the roof displacement are observed for $1.25 \%$ of the inter-story drift threshold. Thus smoothing behavior of reinforcing steel has a minor effect on computed probabilities of the $\mathrm{RC}$ frame. Although the changes in reliability index are not noticeable, but an improvement in the search for design point has observed. It requires $182 \mathrm{~s}$ for reliability analysis with the bi-linear material, where it takes only $78 \mathrm{~s}$ for smooth (M-P) material. Therefore, the maximum trial point distance from the origin is much closer for smooth material than bi-linear material model. This
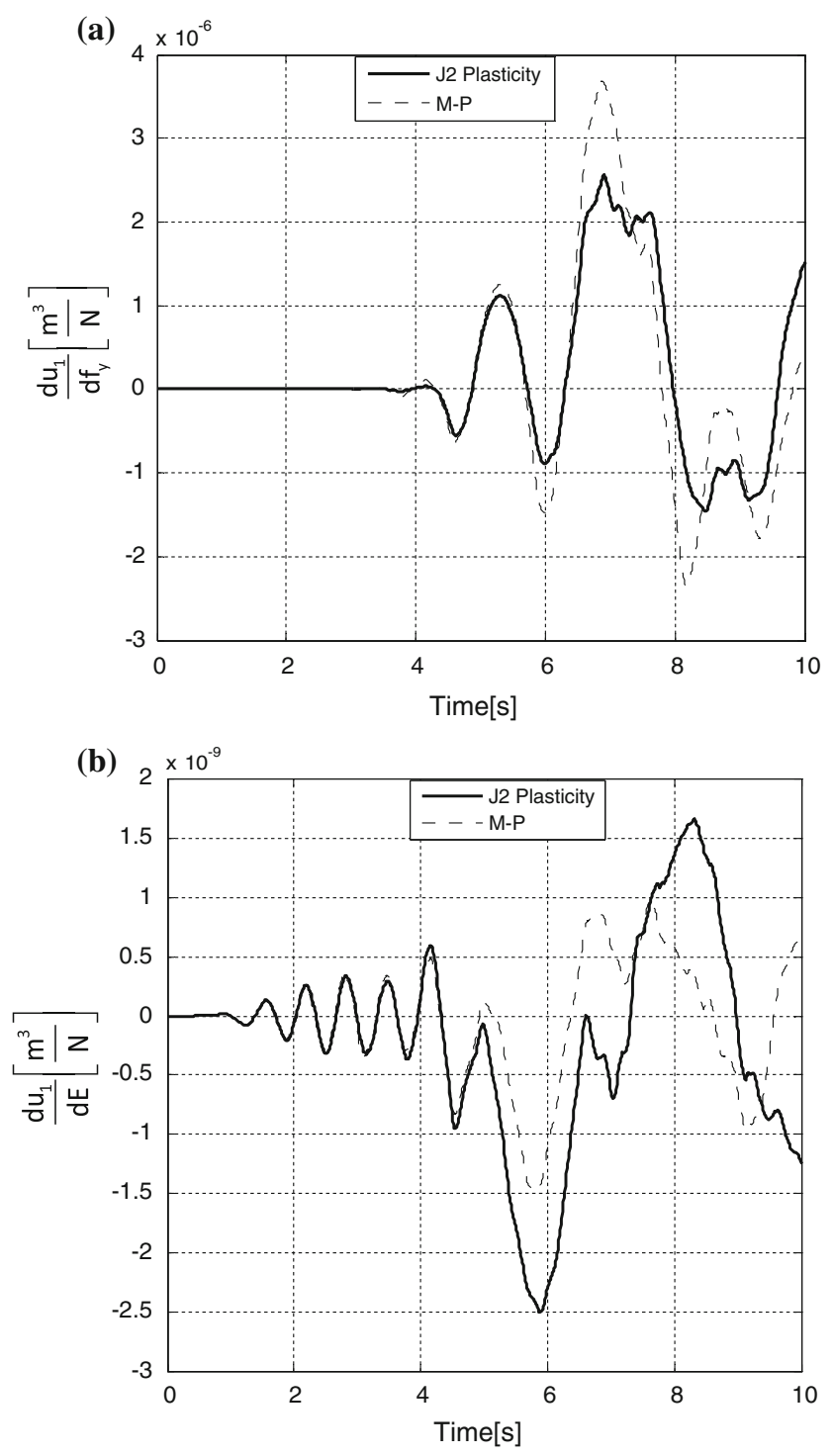

Fig. 12 a Sensitivity of roof horizontal displacement response with respect to $f_{y}$ of steel. b Sensitivity of roof horizontal displacement response with respect to $E$ of steel.

advantage is achieved, because a large portion of the structure exhibits nonlinearity at the mean point by using smooth material model. Thus, using of smooth material model can be a fruitful technique to avoid non-convergence in FE based reliability analysis.

\section{Conclusions}

This work presents the analytical behavior of FE response sensitivities obtained from the different constitutive material models. DDM based nonlinear FE response sensitivity analysis is carried out under static and dynamic loading. FFD method has been used to investigate the accuracy of DDM. Smoothing behavior of both concrete and steel materials has been taken into consideration to evaluate the smoothness effects. After that, structural reliability analysis is performed to observe the smoothing effect of the reinforcing steel on failure probabilities of the frame. The core conclusions of this study can be summarized as follows: 
Table 5 Parameter importance rankings for the inter-story drift of RC frame.

\begin{tabular}{|c|c|c|c|c|c|c|c|}
\hline \multirow{3}{*}{$\begin{array}{l}\text { Response } \\
\text { parameter }\end{array}$} & \multicolumn{4}{|c|}{ Concrete } & \multirow{2}{*}{\multicolumn{3}{|c|}{ Steel }} \\
\hline & \multicolumn{2}{|c|}{ Core } & \multicolumn{2}{|c|}{ Cover } & & & \\
\hline & $f_{c}$ & $\varepsilon_{u}$ & $f_{c}$ & $\varepsilon_{u}$ & $f_{y}$ & $E$ & $b$ \\
\hline$u_{\text {drift }}$ & 4 & 5 & 3 & 6 & 2 & 1 & 7 \\
\hline
\end{tabular}

Table 6 Reliability index $\beta$ for FORM and important sampling of RC frame.

\begin{tabular}{c|c|c}
\hline LSF & FORM & Important sampling \\
\hline \hline$g_{1}$ & 3.19023 & 3.19118 \\
\hline$g_{2}$ & 3.02357 & 3.02583 \\
\hline
\end{tabular}

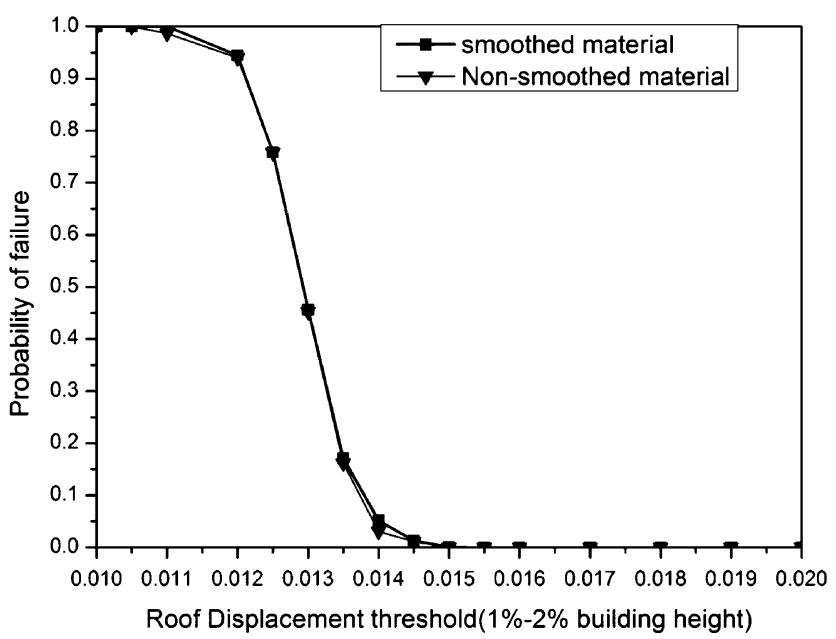

Fig. $13 \mathrm{CDF}$ for roof displacement (threshold varies from 1 to $2 \%$ of building height).

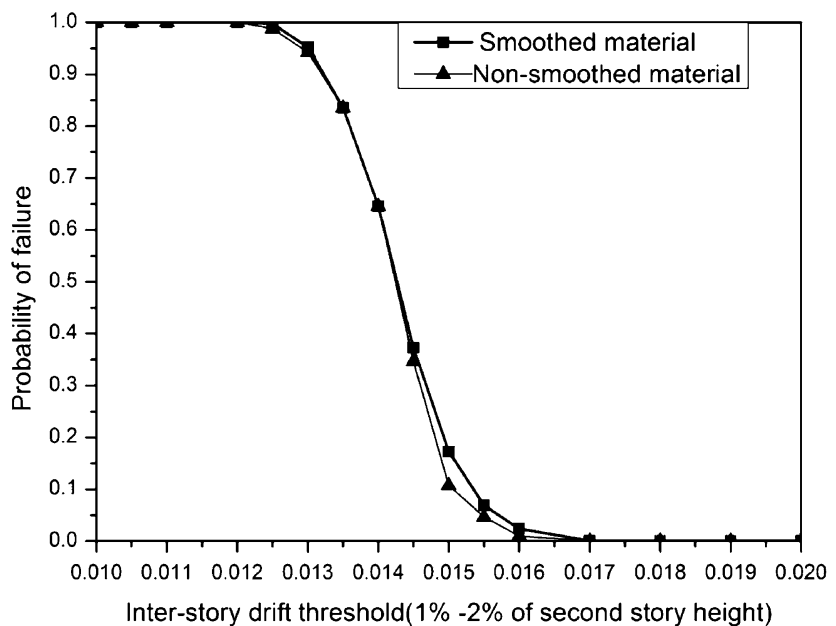

Fig. 14 CDF for inter-story drift (threshold varies from 1 to $2 \%$ of second story height).

1. In quasi-static FE analysis, response sensitivity discontinuities are observed in Popovics-Saenz concrete model without smoothing parameter, Kent-Scott-Park concrete model, and bi-linear steel model. PopovicsSaenz concrete with smoothing parameter and M-P (smooth model) steel models are used to avoid the occurrence of the discontinuity and get the continuous computation of response sensitivity. Therefore, for the design point search, using gradient based optimization algorithms, smooth material model plays an important role to get accurate and efficient computations of response sensitivities.

2. DDM is very accurate and efficient method in computing the response sensitivities and also applicable to any material constitutive model. Good convergence of FFD results with DDM shows the accuracy in computation.

3. Under dynamic motion, sensitivity result of the roof displacement with respect to the core concrete has shown minor effect of smoothing properties for two different concrete models. In dynamic analysis, the displacement response is smooth due to inertia effect of dynamic motion. Linear inertia in the equation of motion also shows considerable smoothing effects on the response sensitivity results of steel constitutive models along the pseudo-time axis. Moreover, a minor discontinuity effect has been found in non-smooth $J_{2}$ plasticity steel along the parametric axis. This effect can be eliminated by using smooth steel model (M-P).

4. Structural reliability analysis shows the influence of smoothing on the computed probabilities of the RC structure. However, more efficient and reasonable reliability estimation can be achieved by using smooth material model compare with bilinear material constitutive model.

\section{Acknowledgments}

This research was supported by the Ministry of Knowledge Economy and Korea Institute of Energy Technology Evaluation and Planning (KETEP) as a part of the Nuclear R\&D Program (No. 20101620100230). The authors would like to express their appreciation for the financial support.

\section{Open Access}

This article is distributed under the terms of the Creative Commons Attribution License which permits any use, distribution, and reproduction in any medium, provided the original author(s) and the source are credited.

\section{References}

Arora, J. S., \& Haug, E. J. (1979). Methods of design sensitivity analysis in structural optimization. AIAA Journal, 17, 970-974. 
ATC-55. (2005). Evaluation and improvement of inelastic seismic analysis procedures. Advanced Technology Council, Redwood City, CA.

Balan, T. A., Filippou, F. C., \& Popov, E. P. (1997). Constitutive model for 3D cyclic analysis of concrete structures. Journal of Engineering Mechanics, 123(2), 143-153.

Balan, T. A., Spacone, E., \& Kwon, M. (2001). A 3D hypoplastic model for cyclic analysis of concrete structures. Engineering Structures, 23, 333-342.

Barbato, M., \& Conte, J. P. (2006). Finite element structural response sensitivity and reliability analyses using smooth versus non-smooth material constitutive models. International Journal of Reliability and Safety, 1(1-2), 3-39.

BSSC. (2003). The 2003 NEHRP recommended provisions for new buildings and other structures. Part 1: Provisions (FEMA 450). Building Seismic Science Council.

Conte, J. P. (2001). Finite element response sensitivity analysis in earthquake engineering. In B. F. Spenser \& Y. X. Hu (Eds.), Earthquake engineering frontiers in the new millennium (pp. 395-401). Lisse: Swets and Zeitlinger.

Conte, J. P., Barbato, M., \& Spacone, E. (2004). Finite element response sensitivity analysis using force-based frame models. International Journal of Numerical Methods in Engineering, 59(13), 1781-1820.

Conte, J. P., Vijalapura, P. K., \& Meghella, M. (2003). Consistent finite-element response sensitivity analysis. Journal of Engineering Mechanics, 129(12), 1380-1393.

Der Kiureghian, A., \& Ke, J. B. (1988). The stochastic finite element method in structural reliability. Probabilistic Engineering Mechanics, 3(2), 83-91.

Ditlevsen, O., \& Madsen, H. O. (1996). Structural reliability methods. New York: Wiley.

Filippou, F. C., Popov, E. P., \& Bertero, V. V. (1983). Effects of bond deterioration on hysteretic behavior of reinforced concrete joints. Report EERC 83-19, Earthquake Engineering Research Center, University of California, Berkeley, CA.

$\mathrm{Gu}, \mathrm{Q}$. (2008). Finite element response sensitivity and reliability analysis of soil-foundation-structure-interaction systems. Ph.D. dissertation, Department of Structural Engineering, University of California at San Diego, La Jolla, CA.

Gu, Q., Barbato, M., \& Conte, J. P. (2009b). Handling of constraints in finite-element response sensitivity analysis. Journal of Engineering Mechanics, 135(12), 1427-1438.

Gu, Q., Conte, J. P., \& Barbato, M. (2010). OpenSees command language manual response sensitivity analysis based on the direct differentiation method (DDM). Berkeley, CA: Pacific Earthquake Engineering Center, University of California.

Gu, Q., Conte, J. P., Elgamal, A., \& Yang, Z. (2009a). Finite element response sensitivity analysis of multi-yield-surface J2 plasticity model by direct differentiation method. Computer Methods in Applied Mechanics and Engineering, 198(30-32), 2272-2285.

Hasofer, A. M., \& Lind, N. C. (1974). An exact and invariant first order reliability format. Journal of Engineering Mechanics, 100(12), 111-121.
Haukaas, T., \& Der Kiureghian, A. (2004). Finite element reliability and sensitivity methods for performance-based engineering. Report PEER 2003/14, Pacific Earthquake Engineering Research Center, University of California, Berkeley, CA.

Hohenbichler, M., \& Rackwitz, R. (1986). Sensitivity and importance measures in structural reliability. Civil Engineering Systems, 3(4), 203-209.

Kleiber, M., Antunez, H., Hien, T.D., \& Kowalczyk, P. (1997). Parameter sensitivity in nonlinear mechanics: Theory and finite element computations. New York: Wiley.

Mahsuli, M., \& Haukaas, T. (2010). Methods, models, and software for seismic risk analysis. In 9th US National \& 10th Canadian Conference on Earthquake Engineering, Toronto, 25-29 July 2010.

Mazzoni, S., McKenna, F., \& Fenves, G. L. (2005). OpenSees command language manual. Berkeley, CA: Pacific Earthquake Engineering Center, University of California.

Menegotto, M., \& Pinto, P. E. (1973). Method for analysis of cyclically loaded reinforced concrete plane frames including changes in geometry and non-elastic behavior of elements under combined normal force and bending. In Proceedings, IABSE Symposium on 'Resistance and Ultimate Deformability of Structures Acted on by Well-Defined Repeated Loads', Lisbon.

Monti, G., \& Nuti, C. (1992). Non-linear cyclic behavior of reinforcing bars including buckling. Journal of Structural Engineering, 118(12), 3268-3284.

Schueller, G. I., Pradlwarter, H. J., \& Koutsourelakis, P. S. (2004). A critical appraisal of reliability estimation procedures for high dimensions. Probabilistic Engineering Mechanics, 19(4), 463-474.

Scott, M. H. (2012). Evaluation of force-based frame element response sensitivity formulations. Journal of Structural Engineering, 138(1), 72-80.

Scott, M. H., \& Filippou, F. C. (2007). Response gradients for nonlinear beam-column elements under large displacements. Journal of Structural Engineering, 133(2), 155-165.

Scott, M. H., Franchin, P., Fenves, G. L., \& Filippou, F. C. (2004). Response sensitivity for nonlinear beam-column elements. Journal of Structural Engineering, 130(9), 1281-1288.

Scott, B. D., Park, R., \& Priestley, M. J. N. (1982). Stress-strain behavior of concrete confined by overlapping hoops at low and high strain rates. ACI Journal, 79(1), 13-27.

Simo, J. C., \& Taylor, R. L. (1985). Consistent tangent operators for rate-independent elastoplasticity. Computer Methods in Applied Mechanics and Engineering, 48, 101-118.

Zhang,Y., Conte, J. P., Yang, Z., Elgamal, A., Bielak, J., \& Acero, G. (2008). Two-dimensional nonlinear earthquake response analysis of a bridge-foundation-ground system. Earthquake Spectra, 24(2), 343-386.

Zhang, Y., \& Der Kiureghian, A. (1993). Dynamic response sensitivity of inelastic structures. Computer Methods in Applied Mechanics and Engineering, 108(1-2), 23-36.

Zona, A., Barbato, M., \& Conte, J. P. (2005). Finite element response sensitivity analysis of steel-concrete composite beams with deformable shear connection. Journal of Engineering Mechanics, 131(11), 1126-1139. 